\title{
On the path to the digital rock physics of gas hydrate-bearing sediments - processing of in situ synchrotron-tomography data
}

\author{
Kathleen Sell ${ }^{1}$, Erik H. Saenger ${ }^{2,3}$, Andrzej Falenty ${ }^{4}$, Marwen Chaouachi ${ }^{4}$, David Haberthür ${ }^{5}$, Frieder Enzmann ${ }^{1}$, \\ Werner F. Kuhs ${ }^{4}$, and Michael Kersten ${ }^{1}$ \\ ${ }^{1}$ Institute of Geosciences, Johannes Gutenberg University, Mainz, Germany \\ ${ }^{2}$ International Geothermal Centre, Bochum, Germany \\ ${ }^{3}$ Ruhr University, Bochum, Germany \\ ${ }^{4}$ GZG Crystallography, Georg August University, Göttingen, Germany \\ ${ }^{5}$ Swiss Light Source, Paul Scherrer Institute, Villigen, Switzerland \\ Correspondence to: Kathleen Sell (sell@uni-mainz.de)
}

Received: 21 March 2016 - Published in Solid Earth Discuss.: 29 March 2016

Revised: 20 July 2016 - Accepted: 22 July 2016 - Published: 22 August 2016

\begin{abstract}
To date, very little is known about the distribution of natural gas hydrates in sedimentary matrices and its influence on the seismic properties of the host rock, in particular at low hydrate concentration. Digital rock physics offers a unique approach to this issue yet requires good quality, highresolution 3-D representations for the accurate modeling of petrophysical and transport properties. Although such models are readily available via in situ synchrotron radiation Xray tomography, the analysis of such data asks for complex workflows and high computational power to maintain valuable results. Here, we present a best-practice procedure complementing data from Chaouachi et al. (2015) with data postprocessing, including image enhancement and segmentation as well as exemplary numerical simulations of an acoustic wave propagation in 3-D using the derived results. A combination of the tomography and 3-D modeling opens a path to a more reliable deduction of properties of gas hydrate-bearing sediments without a reliance on idealized and frequently imprecise models.
\end{abstract}

\section{Introduction}

With the continuous improvement and popularization of high-resolution visualization methods (Holzer and Cantoni, 2012; Desbois et al., 2013; Hemes et al., 2015; Cnudde and Boone, 2013; Liu et al., 2016; Deville et al., 2013; Berg et al., 2013; Pak et al., 2015) digital rock physics gained a firm foothold in the geophysical world as a potent toolset in studies of rock properties (e.g., porosity and permeability) and pore-scale processes like, e.g., electric and heat conductivity or propagation of acoustic waves (Andrä et al., 2013b, a; Madonna et al., 2013; Saenger et al., 2011). In this approach, numerical simulations of transport and petrophysical properties are conducted on realistic rock representations that ultimately can be upscaled and compared to remote sensing data. The advantage of this method over traditional, often oversimplified models lies in a more faithful description of complex pore geometries and microstructures found in natural formations (Andrä et al., 2013b, a). The most common base for the numerical simulation is segmented 3-D reconstructions from various nondestructive $\mathrm{X}$-ray computed tomography (X-ray CT) techniques. With a good tradeoff between the level of detail and investigated sample volume these analytical tools became regularly used on geological samples at ambient or cryogenic conditions (Murshed et al., 2008; Klapp et al., 2012; Pak et al., 2015; Berg et al., 2013; Wang et al., 2015, 2016).

More recently digital rock physics took also on data from a fairly new group of techniques focused on in situ studies recreating complex settings that cannot be easily accessed with conventional means. Two of such difficult environments are certainly marine and permafrost strata that are host to crystalline gas-water compounds known as gas hydrates (GHs) (Sloan and Koh, 2008). Composed predominantly of methane, GHs are a target of intensive geophys- 
ical surveys, drilling and well-logging operations aiming at the identification and quantification of natural deposits of this unconventional source of hydrocarbons (Sloan and Koh, 2008; Boswell and Collett, 2011; Moridis et al., 2011). The identification of potential deposits with remote sensing methods largely relies on high electric resistivity or characteristic seismic anomalies in which the increased velocity of seismic wave is coupled with high attenuations (Best et al., 2010; Guerin and Goldberg, 2005; Matsushima et al., 2015; Priest et al., 2006). The latter phenomenon has been interpreted as losses due to squirt flow in interfacial liquids trapped between mineral frame and GH crystals (Priest et al., 2006) but became confirmed only in recent sub-micrometer CT studies (Chaouachi et al., 2015).

The quantification of GH saturation levels is not straightforward as there is very little known about the formation, microstructure and distribution of hydrates in natural settings, parameters fundamental to the interpretation of geophysical exploration. Different habits, distributions and saturation of gas hydrate crystals in the pore space affect the physical properties of the hydrate-bearing sediment (Priest et al., 2005; Waite et al., 2004). As the recovery of unperturbed natural methane hydrates is very difficult due to their fast decomposition under ambient conditions, a number of researchers have attempted to recreate the natural environment of gas hydrate in sedimentary matrices via laboratory experiments (Berge et al., 1999; Best et al., 2010, 2013; Dai et al., 2012; Dvorkin et al., 2003; Ecker et al., 2000; Hu et al., 2010; Li et al., 2011; Priest et al., 2006, 2009; Spangenberg and Kulenkampff, 2006; Yun et al., 2005; Zhang et al., 2011). This collective effort eventually leads to a set of idealized micro-structural models (Fig. 1), but the approximations turned out to be still far from being satisfactory. None of the simplified models could accurately predict GH saturations from field electric resistivity or seismic data alone (Waite et al., 2009; Dai et al., 2012). This might now change with the advent of high-resolution in situ X-ray CT methods that open up new a possibility to explore various nucleation and growth paths of GH in a sedimentary matrix in three dimensions with a pixel resolution below $1 \mu \mathrm{m}$. The application of digital rock physics to such complex systems is certainly non-trivial due to a number of constraints imposed by the experimental setups (e.g., fixed sample-detector distance, high attenuation of the X-ray beam, low contrast), but it still remains a viable way to improve the quantification of $\mathrm{GH}$ contents and to characterize the properties of the system.

The primary challenge is found in the acquisition of highquality 3-D models that are capable of capturing fine features such as micro-cracks, fine porosity and grain-to-grain contacts that could potentially influence the computed properties (Kerkar et al., 2014; Chaouachi et al., 2015). Another critical point specific to the seismic anomaly in GH sediments is a clear identification of the at most a few-micrometers-thick water film separating mineral frame and GHs. As we demonstrated in our recent work (Chaouachi et al., 2015; Falenty et

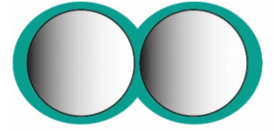

A) Encrustation

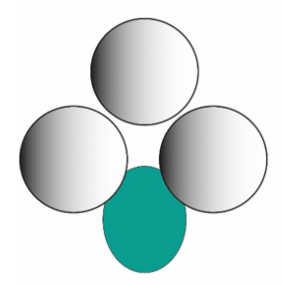

$\begin{array}{lll}\text { C) Matrix-supporting } & \text { D) Pore-filling }\end{array}$
Figure 1. Various distributions of how hydrate might be located in the sedimentary matrix as discussed in previous studies: encrustation, cementation, matrix supporting and pore-filling (modified after Dai et al., 2004).

al., 2015) these high requirements can be met by synchrotron sources due to their unmatched resolution, high photon density and tunable energy of the beam. Yet, even in this case the semi-automatic segmentation and labeling is a difficult task due to a low density contrast and refractory edge enhancement. In this study, we present a workflow for the image enhancement, segmentation and labeling of these data for further 3-D modeling.

\section{Experimental setup and data acquisition}

In the following section the in situ experiment and scanning procedure will be described briefly as details are given in Chaouachi et al. (2015) and Falenty et al. (2015). As the nucleation and formation of hydrates within the pore space occurs on the nano- to microscale, high-resolution synchrotron radiation X-ray tomography (SRXCT) was the best experimental choice.

\subsection{In situ experiment}

SRXCT scans were obtained at the TOMCAT beamline of the Swiss Light Source (SLS), Paul Scherrer Institute (PSI) in Villigen, Switzerland (Stampanoni et al., 2006). The high brilliance, flexible selection of the energy window and exceptional resolution of this setup allows for studies of dynamic processes in complex environmental cells with an excellent signal-to-noise ratio and fast acquisition time (Fig. 2a). The formation process was observed in a custom-built in situ cell composed of (1) an aluminium base, (2) sample holder mounted on top of the base and (3) TECAPEI (polyetherimide) dome used to seal the setup (Fig. 2b). Temperature control is provided by a Peltier element mounted with the cold side at the bottom of the aluminum base. The temperature of the sample is actively controlled via a PID controller 
with long-term stability within $\pm 0.1^{\circ} \mathrm{C}$. In order to avoid potential vibrations from a pump the hot side of the Peltier element is cooled with a laminar gravity-driven flow of water. The pressure inside the cell is measured with an ASHCROFT KXD pressure sensor and the data were recorded every $5 \mathrm{~s}$ on a computer. The sample holder with a wall thickness of $0.1 \mathrm{~mm}$, an inner diameter of $2 \mathrm{~mm}$ and a length of $10 \mathrm{~mm}$ is made also out of aluminum alloy, which ensures a good thermal conductivity to lower the temperature $T$ to a constant of $T=2{ }^{\circ} \mathrm{C}$. The size of the sample holder was chosen to fulfill the size requirements of the numerical modeling planned thereafter but also to meet the limitations concerning beam energy attenuation (Fig. 2b). Using methane gas would lead to considerable additional challenges because of high required gas pressures during the measurements and a more difficult data processing due to a low density difference between water and methane hydrate (Jin et al., 2006). In order to mitigate these complications, in our experiments methane was substituted with xenon (Xe) gas, known to be equivalent with respect to the resulting gas hydrate in all important physical properties; xenon hydrate forms at very moderate $p$ - $T$ conditions of $T=2{ }^{\circ} \mathrm{C}$ and a very moderate pressure of $p \geq 0.2 \mathrm{MPa}$. The ambient atmosphere in the closed volume is replaced via several compression and decompression cycles with pure Xe gas while staying below the thermodynamic stability boundary of Xe hydrate (Chaouachi et al., 2015). In this study, the focus is on samples containing natural quartz sand of about $200-300 \mu \mathrm{m}$ grain size. Details on this sedimentary material can be found in Chuvilin et al. (2011).

\subsection{Scanning procedure}

For each tomogram, 3201 (or 1501) projections at an integration time of $150 \mathrm{~ms}$ (or $350 \mathrm{~ms}$ ) each were acquired over a sample rotation of $180^{\circ}$ with a monochromatic Xray beam energy of $21.9 \mathrm{keV}$. After penetrating the sample, X-rays were converted into visible light by a $20 \mu \mathrm{m}$ thick scintillator LuAG:Ce and captured by a high-resolution CCD camera of $2048 \times 2048$ pixels. Two different objectives (UPLAPO10 $\times$ or UPLAPO20 $\times$ ) with an optical 10fold or 20-fold magnification were used, respectively. The data have been reconstructed out of cam by a gridded Fourier transform-based algorithm (Marone and Stampanoni, 2012). The reconstruction process yields in an image matrix of $2560 \times 2560 \times 2160$ voxels, with an isometric voxel size of 0.74 and $0.38 \mu \mathrm{m}$ at 10 -fold and 20 -fold magnification, respectively. Time-resolved scanning was achieved by starting the reaction, stopping it after a prior lab-tested reaction time by reducing pressure to the stability boundary, with allowance for the system to stabilize, followed by subsequent scanning (Chaouachi et al., 2015; Falenty et al., 2015). This procedure is called "stop-and-go in situ tomography", which was repeated multiple times to follow consecutive stages of hydrate formation.

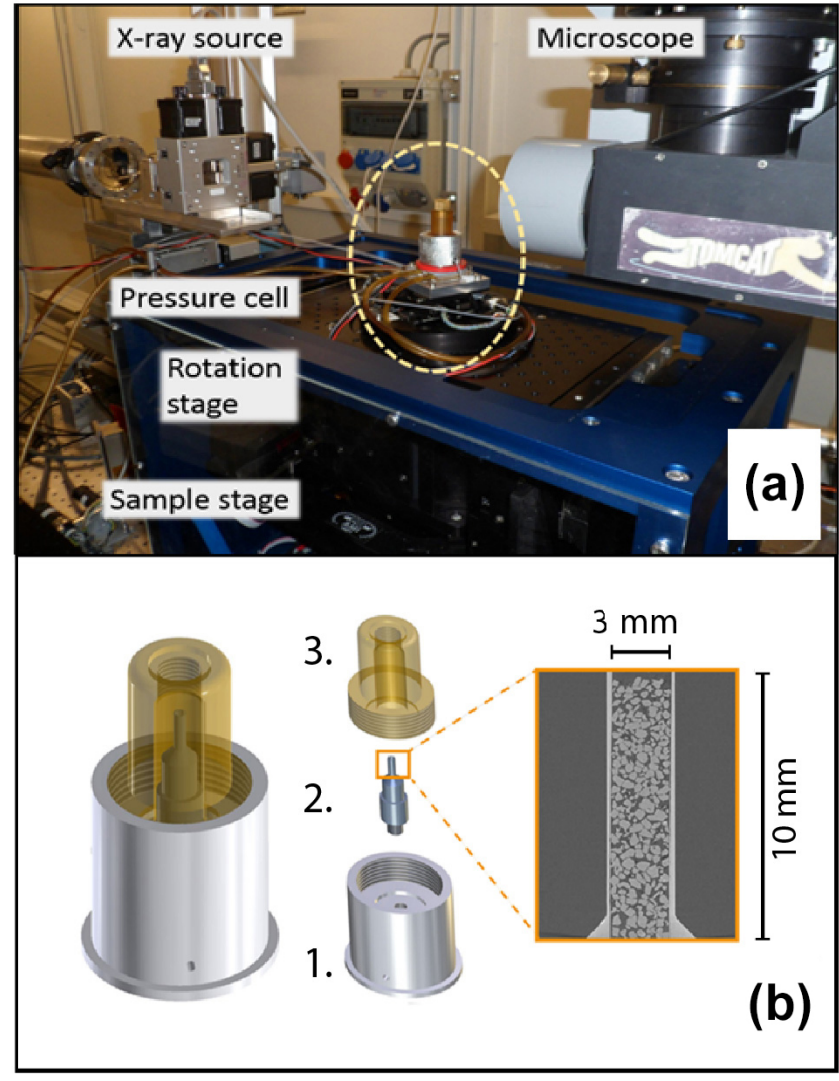

Figure 2. (a) Experimental setup at the TOMCAT beamline. (b) The pressure cell consisting of the base (1), the sample holder (2) and the dome (3).

\section{Data processing}

\subsection{Reconstruction}

To translate the sinograms to 3-D tomographic images the regridding reconstruction algorithm (Marone and Stampanoni, 2012; Stampanoni et al., 2006) was used. The reconstructed tomograms revealed that in spite of contrast enhancement with Xe gas the grey value differences between the gas and water phase were distinguishable by eye but too low for further segmentation approaches. Moreover, a correct visualization of the thin fluid layer located in between the grains and the hydrate (Chaouachi et al., 2015) required a new approach to enhance the image contrast. For this, the original projections of a scan were recreated to obtain the original sinograms. Following the refraction index of the Henke database with water as the starting value (Henke et al., 1993) for a beam energy of $21.9 \mathrm{keV}$, the material's refractive index deviates from unity by the real part $\delta=4.808 \times 10^{-7}$ and by the imaginary part $\beta=2.563 \times 10^{-10}$. The Paganin algorithm (Paganin et al., 2002) is able to extract phase information in samples, which was tested on the present data to recover contrast between the xenon and the aqueous phase. 

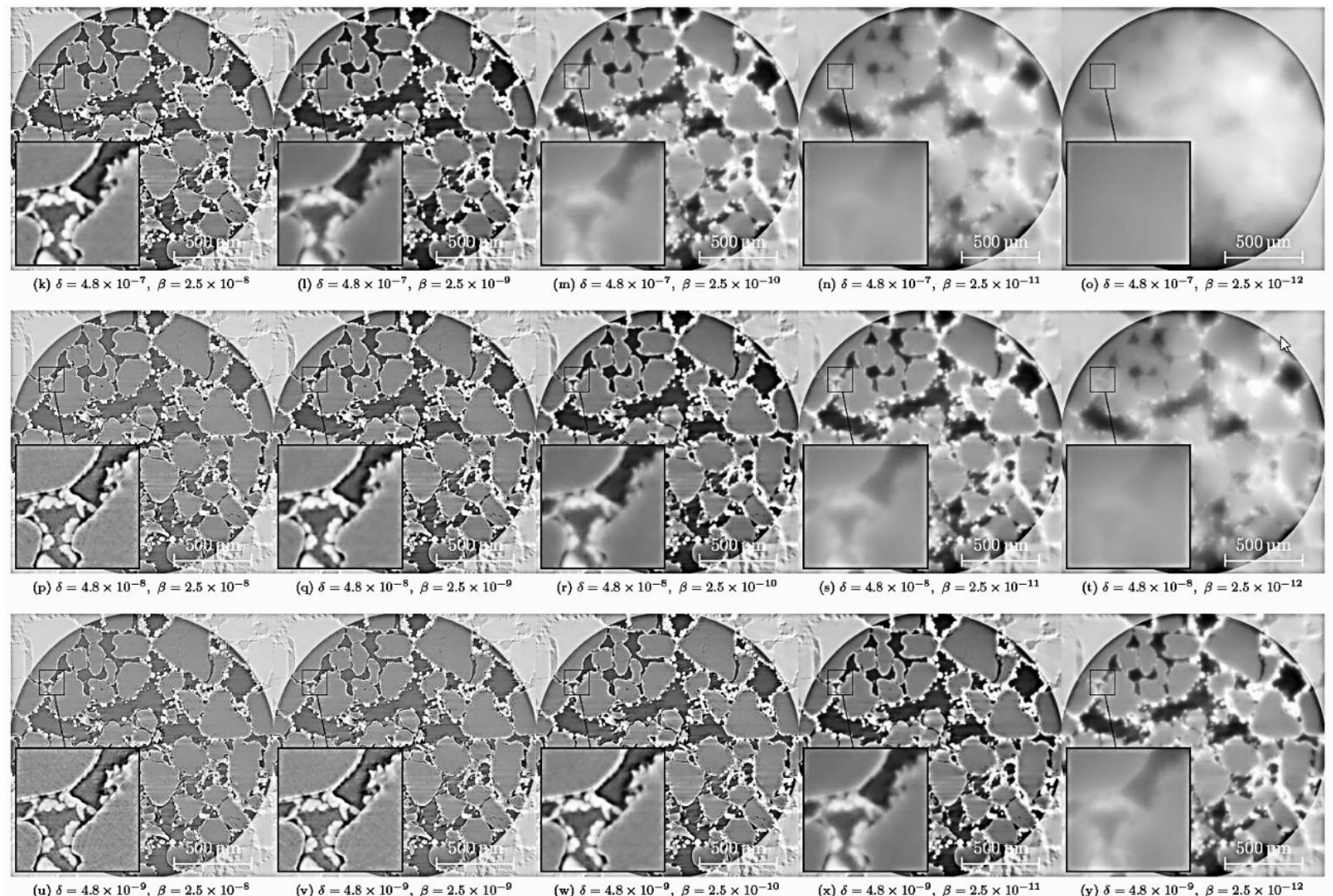

Figure 3. Selected results of the approach to enhance the contrast between the gaseous and aqueous phase by applying the reconstruction algorithm of Paganin on a $2560 \times 2560$ voxel 2 -D slice. The insets show a $75 \times 75$ pixel size region of interest, magnified $5 \times$.

A representative sample has been chosen to apply the Paganin iteration. For this test, the sample to detector distance was set to $25 \mathrm{~mm}$ and the rotation center to 1281.2. In total, 25 different parameters were chosen and applied to various 2-D slices. The reconstruction for each set of parameters did not meet the expectations to show a large and clear border between Xenon and water (Fig. 3).

\subsection{Image enhancement}

At this point the reconstructed images are limited to the spatial resolution and affected by image artifacts but already enable one to distinguish between gas, water, sediment grains and hydrate. Image artifacts that commonly occurred in the samples were inhomogeneity in grey values, streaks resulting from bad rotation alignment and edge enhancement.

The first step in data post-processing is to reduce image noise and scan artifacts using common image filters, calibrated for the appropriate dimensions and kernel window sizes (Sell et al., 2015). The software package Avizo 7.1 (FEI, France) has been used for the steps of image filtering and segmentation. One must be aware that every step of image enhancement changes the original dataset affect- ing subsequent steps required for data analysis (Sell et al., 2013b). Working on big datasets often stresses the computational power to an extent where an effective processing is not accomplishable anymore. Therefore it is highly recommended to split the datasets into smaller sub-volumes before taking further action of data post-processing. It is essential to deploy the same enhancement steps and parameters for each digitally sampled sub-volume. Avizo offers several options including the extract subvolume function, the split volume function and the region of interest cropping tool to gain smaller sub-volumes. The cropping tool is not recommended for the described situation as it substitutes the complete dataset by the selected region of interest instead of gaining several smaller volumes in one attempt. For this study, the extract subvolume function was the best choice. As the full datasets each have a known size of 28 GB equal to $2560 \times 2560 \times 2160$ pixels it was decided to split the dataset to eight sub-volumes each of a size of $1280 \times 1280 \times 1080$. Subsequently the sub-volumes are rated in terms of image artifacts by considering various slices in $x-y, x-z$ and $y-$ $z$ direction. Choosing the right image enhancement technique might require an extensive testing of the available filters prior 


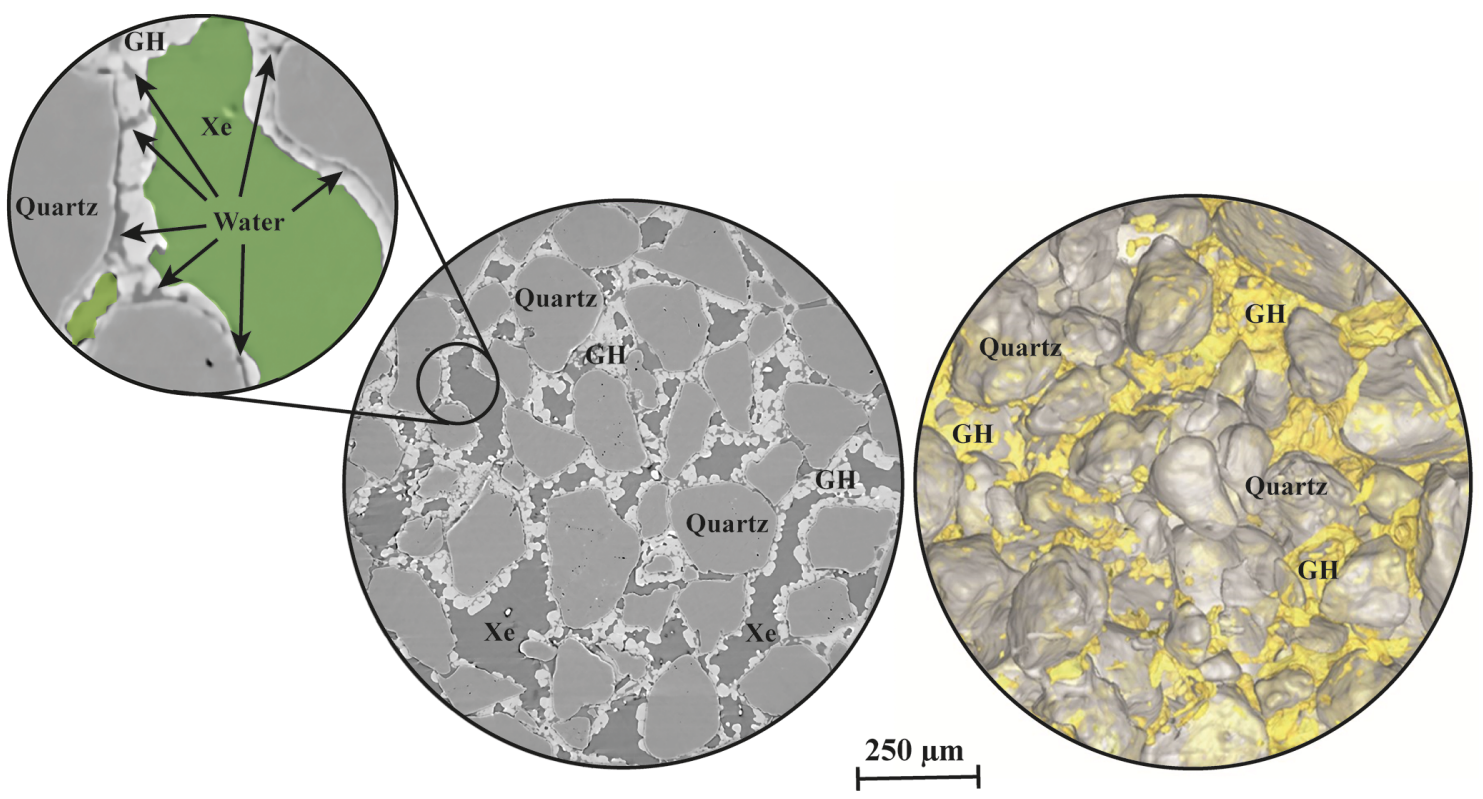

Figure 4. Overview of a 2-D slice in X-Y direction and the equivalent 3-D rendered volume. The presented sample contains 17 vol $\%$ hydrate. The 2-D slice has a dimension of $2560 \times 2560$ pixels with a pixel size of $0.76 \mu \mathrm{m}^{2}$. The volume-rendered image depicts the quartz grains in grey and the hydrate in yellow - this dataset is suitable for further model investigations. The zoom-in depicts all the phases present in the samples: xenon - Xe (green), quartz grains, gas hydrate - GH and water.

to further steps. Depending on the dataset and the characteristics of the image artifacts and noise, filters have different impact on the data. Therefore, as we operate at the limiting edge of gaining image quality and running into data bias, considerable caution must be exerted when treating this multi-phase system.

\section{Pre-analysis of image enhancement}

A study on the effect of various image filters on modeling result was necessary to determine the best option of filters aiming at high-quality images to gain volume-rendered images (Fig. 4). For the pre-study well-known and established filters from former case studies (Sell et al., 2013a; Madonna et al., 2013; Shulakova et al., 2013) have been applied to a region of interest $(400 \times 400 \times 400$ voxels $)$ cropped from the original raw dataset. Five datasets which derived from samples containing approximately $17 \mathrm{vol} \%$ of hydrate (free pore water is fully transformation to hydrate) were selected. The knowledge on the hydrate bulk in the samples will be used as a proxy later. In the following we provide a list of the applied filters and settings used in this study.

The concept of the anisotropic diffusion filter (AD) filter is to smooth out noise in predefined areas of an image, but stopping at sharp edges representing boundaries between phases. This way, edges and sharp boundaries between phases are preserved, and image noise is significantly reduced (Kaestner et al., 2008; Porter and Wildenschild, 2010). A comparison of the current voxel with the grey values of its six neighbors takes place, and diffusion is fulfilled when the threshold stop criterion is not exceeded. If the difference between one voxel and its six adjacent neighbors exceeds the given value no diffusion takes place. Another option to control the diffusion process of the filter is to reduce or increase the diffusion time. The parameter number of iterations defines how often the algorithm will be used on the data. The bigger this number is, the more blurred is the resulting image. Smoothing is performed by applying a Gaussian filter. For our investigations the threshold stop criterion was set to the value 22968 as this is the approximated transition of the grain phase to hydrate. $\mathrm{AD}$ was run on $\mathrm{CPU}$ device with five iterations. The adaptive histogram equalization filter (AHE) performs a so-called contrast limited adaptive histogram equalization (CLAHE) on the dataset. The CLAHE algorithm in Avizo partitions the images into contextual regions and applies the histogram equalization to each one. This evens out the distribution of used grey values and thus makes hidden features of the image more visible (Reza, 2004). For this study the brightness was set to 0.716 , the contrast to 0.41 and the clip limit to 7 after trying various values ahead to gain a reasonable result. The edge detection moments filter (EDM) is a statistical feature detection to mask out noisy regions or for edge detection. Here, the brightness was set to 0.5 and contrast was set to 2. The edge detection Sobel filter (EDS) filter is based on the Sobel operator which preserves grain boundaries by searching for the most homogeneous feature of the input data and assigns the averaged value to an elementary volume where it highlights boundaries between different materials (Shulakova et al., 2013). For this study, the EDS was 


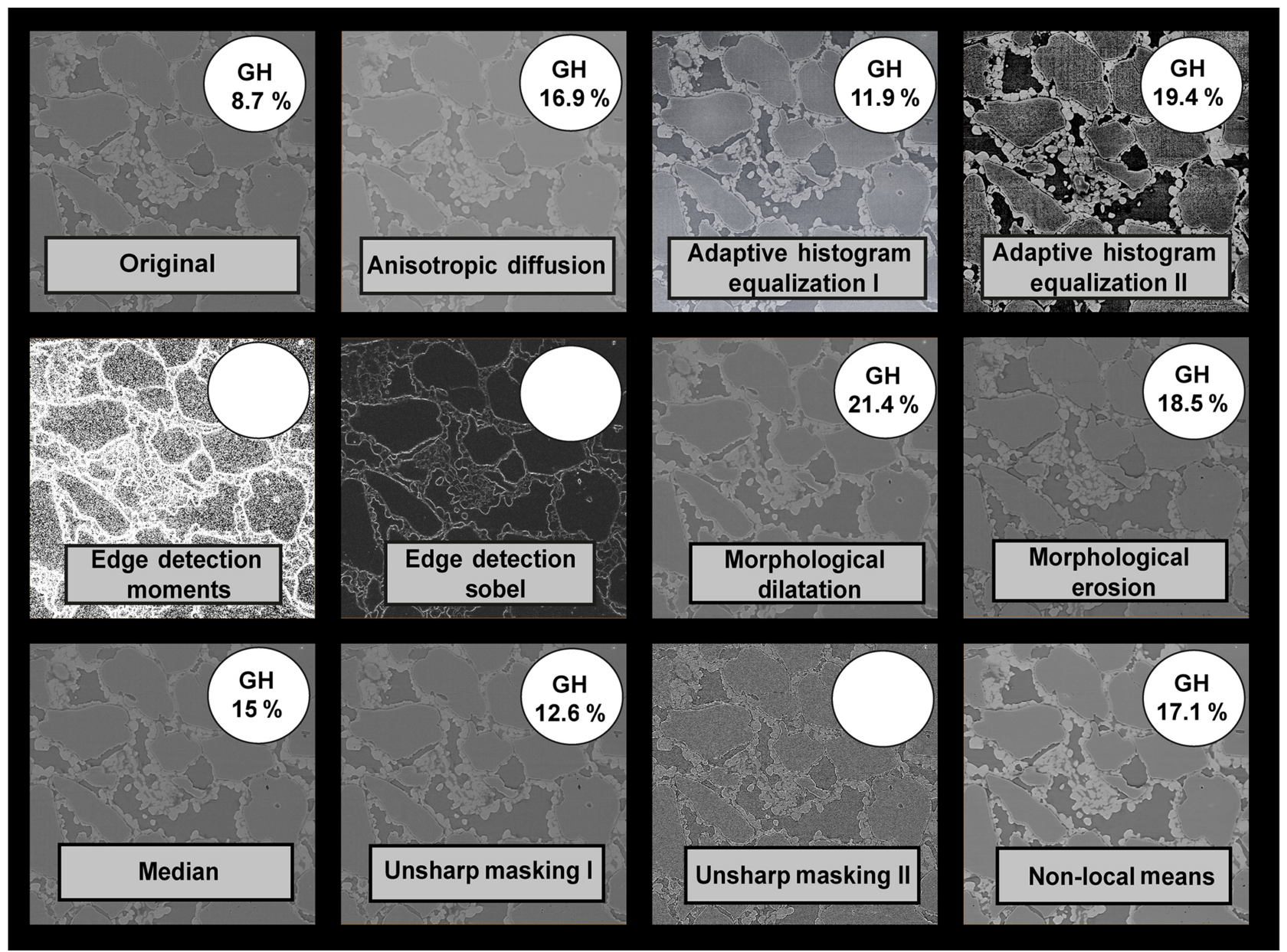

Figure 5. Selected filters applied to the original image to enhance the quality for further segmentation steps. Also, the results of the numerical estimation of hydrate content with the PoroDict module of GeoDict after arithmetic correction are depicted in the upper right corner. Blank circles indicate poor image quality where segmentation was not accomplishable.

set to a brightness of 0.639 at a contrast of 2 . The morphological dilatation filter (MD) noise reduction filter was applied to replace the value of a pixel by the largest value of neighboring pixels. The shape of the neighborhood can be defined via the input field neighborhood, which was set to 8 . The morphological erosion filter (ME) filter works contrary to the MD by replacing the value of a pixel by the smallest value of 8 neighboring pixels. The 3-D median filter $(\mathrm{M})$ is a simple edge-preserving filter which was set to a kernel size of 3 . This filter calculates the mean grey value of neighboring voxels, in our case 9 since the filter was executed in 3-D mode. Subsequently, the initial voxel is replaced by the resulting mean grey value. The unsharp masking filter (UM) sharpens an image using an unsharp mask. In the UM approach for image enhancement, a weighted fraction of the high-pass-filtered version of the image, which results in the unsharp mask, is added to the original image (Ramponi, 1999). The unsharp mask is computed in Avizo by a Gaussian filter of a certain kernel size. For this study the UM was applied with different sharpness settings of 0.5 for UM1 and 1 for UM2 at a kernel size of 3. The non-local means filter (NLM), implemented in Avizo, is a windowed version of the non-local means algorithm (Buades et al., 2005a, b). The main aim is to denoise data based on comparing voxels for similarities in a selected window in which a new weight for a voxel is assigned. After a Gauss kernel was run on the weighted values, the new value will be assigned replacing the former grey values. The filter is most efficient if the image is affected by white noise. In Avizo the parameter window size, the local neighborhood and the similarity value can be customized. The NLM filter is also an appropriate tool for salt-and-pepper denoising, which is caused by image sensor defects (Sarker et al., 2012). In this study, the NLM filter was run in 3-D mode on CPU device. The search window was equal to 21 and the local neighborhood set to 6 at a similarity value of 0.71 .

Adjacent, all filtered datasets were segmented using the same routine which will be described in the following section "Segmentation and volume rendering". For this, binary 


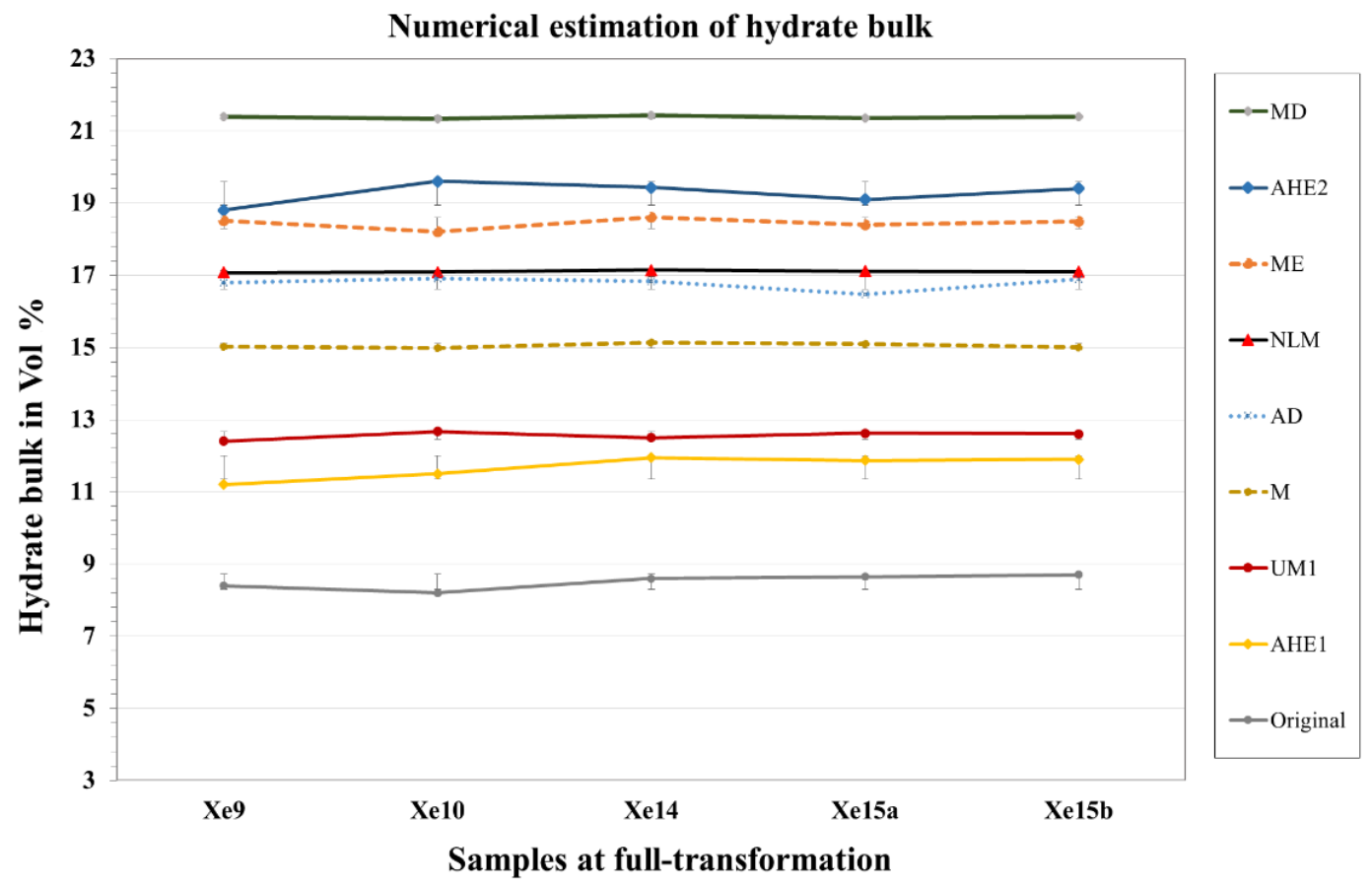

Figure 6. Diagram of the results obtained from numerical estimation of the hydrate content in five different samples containing approx. $17 \%$ controlled by the water available at the initial formation stage.

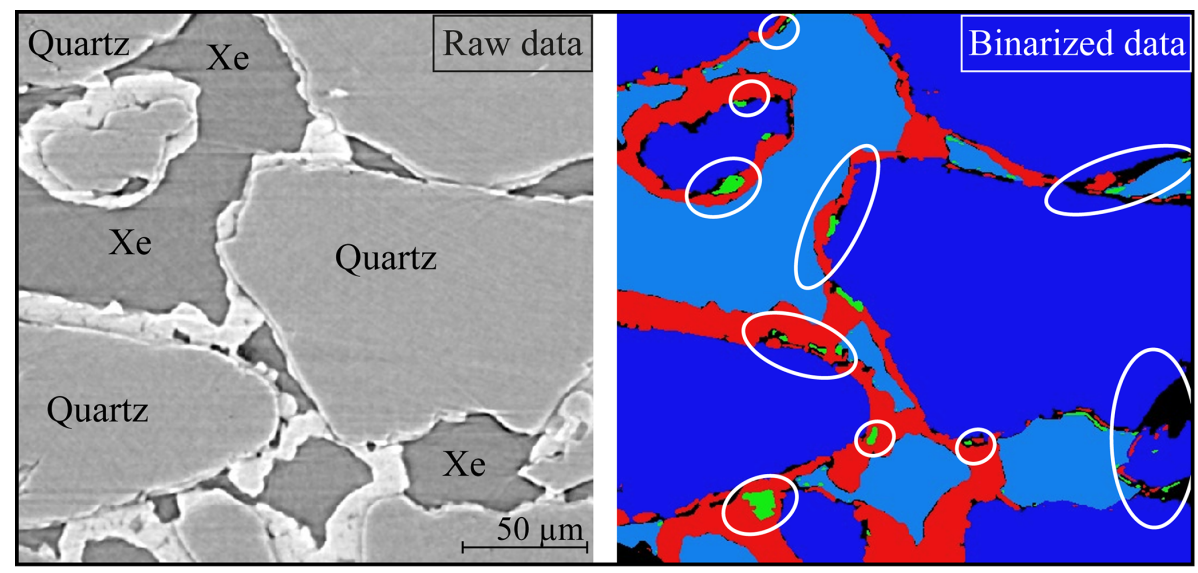

Figure 7. Segmentation problems occurring in samples of low-grade image enhancement quality.

values of 0,1 and 2 were assigned to the pore space, grains and hydrate phase, respectively. Three of the nine filtered volumes have not been binarized as the necessary segmentation routine was not applicable to result in datasets intended for numerical investigation. The module PoroDict of the software package GeoDict (Math2Market) was executed on the binarized images to quantify the hydrate in the filtered datasets. Even though the effects on image quality may not be obvious at first glance, further numerical quantification of the hydrate phase revealed significant differences (Fig. 5).

In Fig. 6 all results regarding the image filter run on five representative volumes are depicted. According to the nu- merical results of the described preliminary study, the best fits to perform image enhancement were the anisotropic diffusion filter $\left(\bar{X}_{\text {hydrate }}=16.79 \%\right)$ and the non-local means filter $\left(\bar{X}_{\text {hydrate }}=17.11 \%\right)$, since both show good agreement in consistency and subsequent numerical estimation of the hydrate bulk. The final decision was to use both filters in a combined manner on the datasets in 3-D mode, which arose because neither the AD filter nor the NLM filter worked on all phases properly. The AD filter caused problems when targeting the quartz-gas phase boundary and the NLM filter hampered the segmentation of the quartz-hydrate phase boundary. Both edge detection filters, Sobel (EDS) and oments 


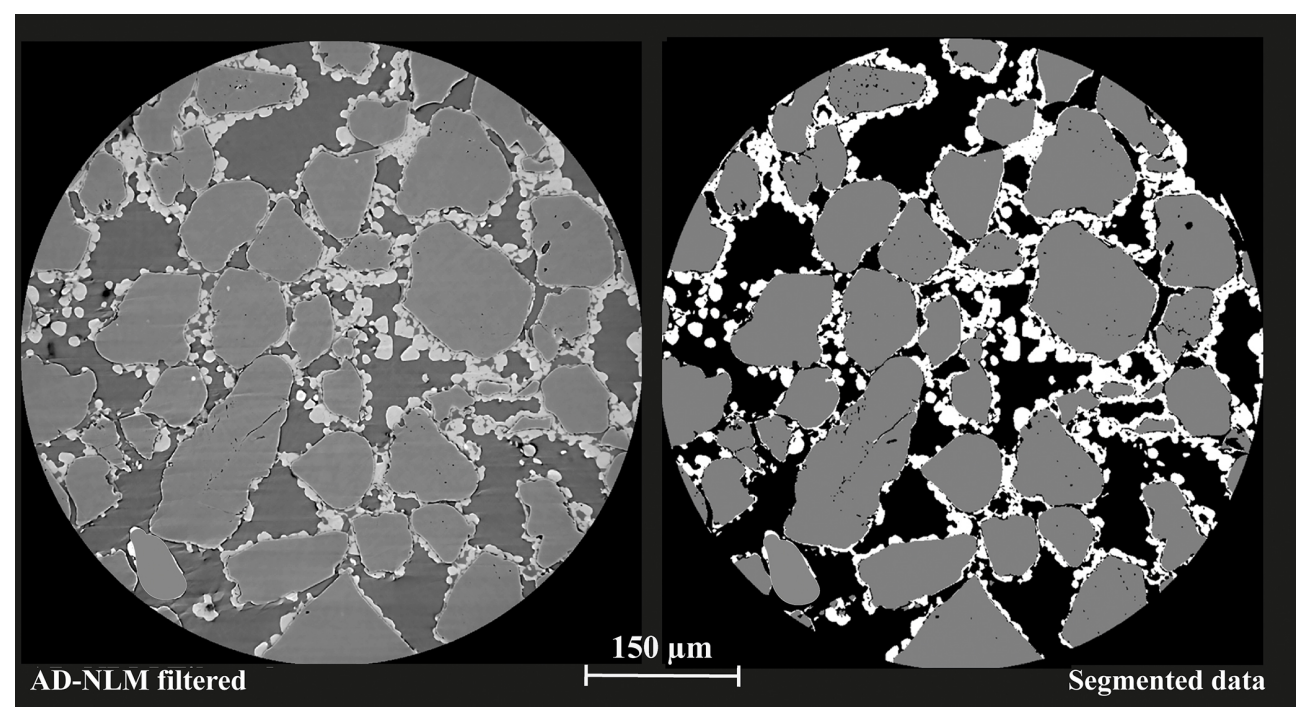

Figure 8. Example on the right is of a successfully segmented dataset. On the left a representative 2-D slice in X-Y direction and the resulting binarized image. Size of the 2-D slice is $2560 \times 2560$. Black - pore space, grey - quartz and white - hydrate. This segmented data are ready to be utilized as a direct model input.

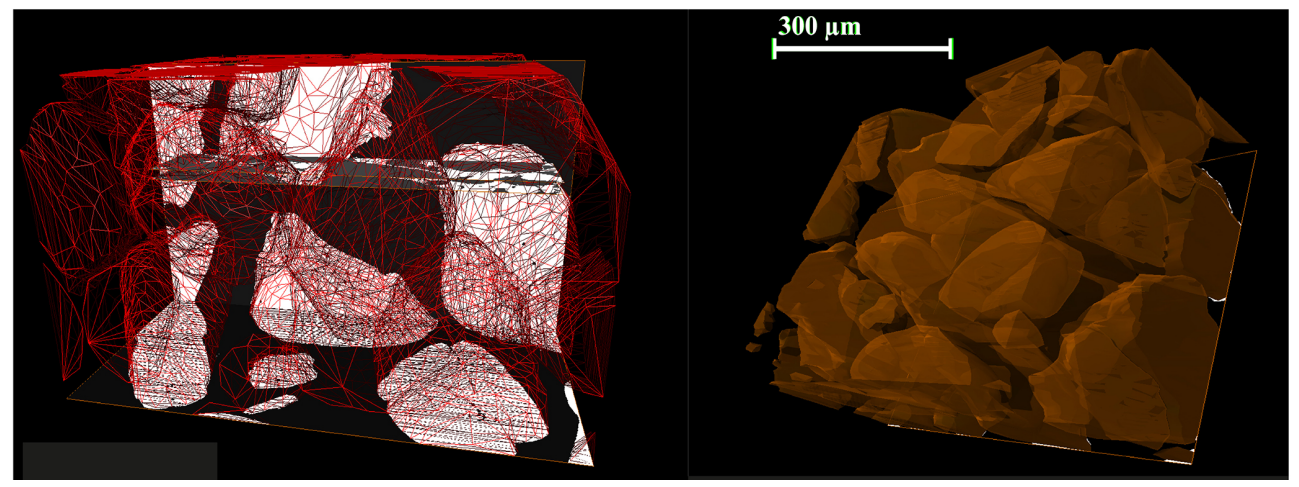

Figure 9. Surface triangulation of quartz grains and the surface view on $\alpha$-scale transparency.

(EDM), were not suitable for the data since the subsequent segmentation could not be carried out.

\subsection{Segmentation and volume rendering}

In this section, we describe the necessary steps of segmentation for this study. Those steps are crucial if the obtained 3$\mathrm{D}$ data will be used further as a numerical model input. This process results in the transformation of voxels of certain grey scale range. Given that a histogram-based segmentation was impossible to conduct, we combined the watershed algorithm and a region growing technique to classify features like phase distribution (Sell et al., 2015) in the filtered sub-volumes of every full dataset (each $28 \mathrm{~GB}$ ). Watershed algorithms are used to semi-automatically segment tomographic data, treating grey values of the gradient image as a topographic map where each grey value stands for a specific altitude. After the placement of seeds or marker regions in catchment basins representing minima, which are basically homogeneous areas with a low gradient, the image is virtually immersed. As soon as "water" from different basins meets, a so-called watershed boundary is placed in between (Vincent and Soille, 1991; Wang, 1997). The algorithm performs well, placing watersheds on sharp intensity edges showing steep gradients and producing maxima in the gradient image as discussed in detail in previous literature (Iassonov et al., 2009; Bleau and Leon, 2000). With this approach it was possible to extract the hydrate phase and the gas-water phase. The quartz phase caused major problems because at the rims of the grain appeared strong irregular edge enhancement effects that could not be removed by filtering (Fig. 7).

As the enhanced edges were of almost the same grey value as the hydrate phase, masking was applied. First, the void and the hydrate were segmented using the watershed algorithm; then the resulting binarized volume of two phases was used to mask the original data in order to extract the quartz 

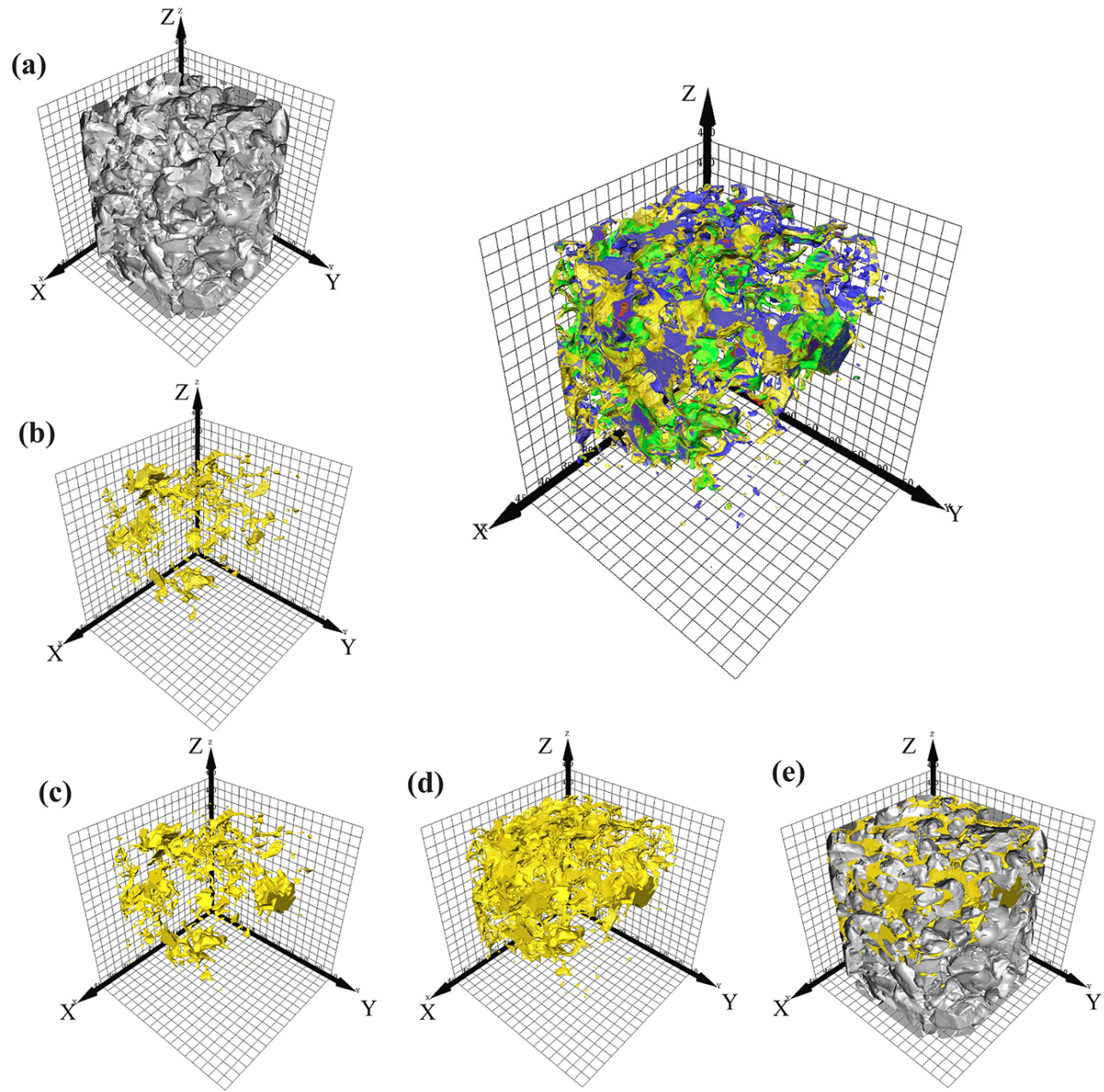

Figure 10. Series of hydrate formation visualized in 3-D. For this a region of interest of $400 \times 400 \times 400$ was picked: (a) initial state where no hydrate is present; (b) and (c) hydrate formation starts and proceeds from a free-gas environment; (d) full-transformation state; (e) fulltransformation state embedded in the sedimentary matrix. The figure in the center shows all hydrate formation steps within one image where some steps are set on $\alpha$-scale transparency to gain information on hydrate structures beneath.

phase, which worked pretty well. Several arithmetic corrections including numerical reconstruction steps and morphological dilatation and erosion were performed to split phases before resulting in a satisfying binarized dataset. Initially every filtered dataset was segmented into three classes: water of gas-filled pores, quartz and hydrates. A fully binarized dataset along the AD-NLM filtered data is shown in Fig. 8. For the purpose of 3-D visualization, we recommend to create a triangulated surface of the binary data (Fig. 9) followed by the application of volume rendering (Fig. 10). For this we used the unconstrained smoothing algorithm and the compactify function available in Avizo.

Due to the earlier mentioned low-density contrast issue between the gaseous and aqueous phase the segmentation of the residual water film was accomplished slice by slice for smaller regions of interest using a region growing function
(Fig. 11). For visualization in 3-D a surface creation tool is applied prior the actual rendering.

\subsection{Pre-analysis of the effect of image enhancement on numerical simulations}

Note that the inability to fully characterize the microstructural details of the material under examination can lead to disagreements between numerical estimates of mechanical properties based on SRXCT images and experimentally derived results (Sell et al., 2013b). Such microstructures are thought to significantly influence the effective elastic properties of a rock sample. Performing such study directly on the hydrate-bearing sediments would have taken too many side effects into account as described earlier (including strong Xray attenuation, liquid and hydrate phases). Consequently, we have decided to perform a pre-analysis to predict the effect of 


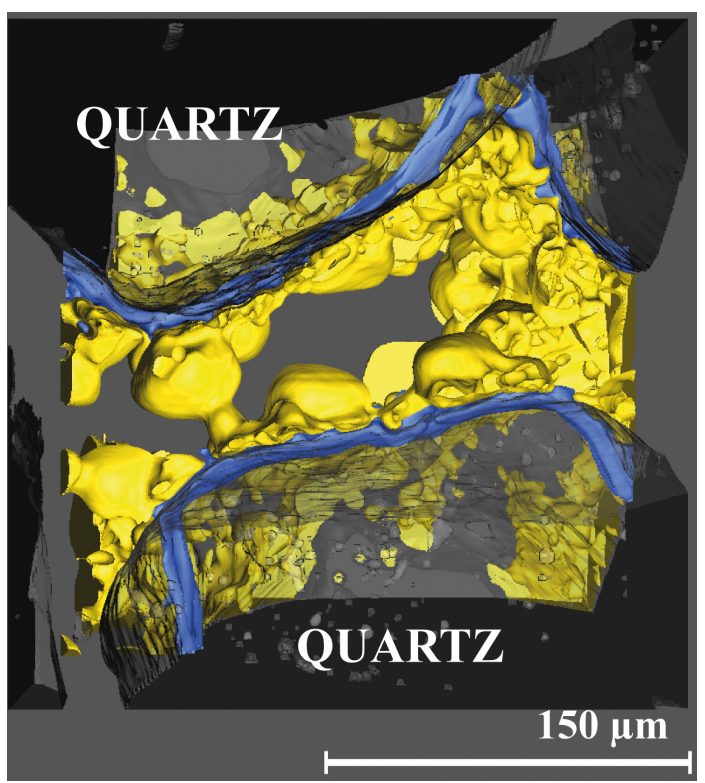

Figure 11. Volume-rendered image of the water film (blue) in between hydrate (yellow) and the quartz grains - for better understanding the outer surface of the grains - is depicted at $\alpha$-scale transparency. Voxel resolution for this scan was $0.38 \mu \mathrm{m}$.

image enhancement on subsequent modeling based on synchrotron data of a nearly one-phase material. The pre-study was done on a synchrotron scanned Berea Sandstone dataset, a well-known "standard" in CT studies and lab investigations (Andrä et al., 2013b, a). We chose a cylindrical specimen of $3 \mathrm{~mm}$ diameter, which was also scanned at the TOMCAT beamline of the Swiss Light Source at the Paul Scherrer Institute in Villigen, Switzerland. The reconstruction process yielded an image matrix of $1024 \times 1024 \times 1024$ voxels, with a voxel size of $0.74 \mu \mathrm{m}$ resulting from the selected 10 fold magnification and a field of view of $1.5 \times 1.5 \mathrm{~mm}$. Also in this case artifacts from the scanning and reconstruction process have been found (e.g., noise, streaks, non-uniform brightness and edge enhancement). These have been treated as discussed in the section "Image enhancement". For further investigations a region of interest called Ber1 cropped to $400 \times 400 \times 400$ voxels was chosen from the open-source dataset case 3, which can be downloaded from supplementary information of Madonna et al. (2013). The 3-D median filter has been applied to Ber1a, the non-local means filter to Ber1b and the anisotropic diffusion filter to Ber1c. All datasets including the original unfiltered have been segmented using the semi-automatic watershed algorithm. Further numerical investigations were conducted including the determination of the total porosity Phi, the permeability $K$ and the effective $P$ wave velocities. Undoubtedly the variation in image enhancement and subsequent determination of grain contact has a strong effect on the obtained rock properties.
The results showed that the effect of image enhancement leads to significant variation regarding the assigned grain contacts (Fig. 12). The total porosities vary from $12 \%$ for the unfiltered dataset Ber1 to $18 \%$ for the dataset Ber1b where the image noise was reduced by applying the NLM filter. Hence the majority of the pores $(>98 \%)$ was found to be connected on the basis of the open-closed porosity approach of the PoroDict module of GeoDict. The watershed algorithm applied on the samples (Ber1a-c) had influence on neither the porosity nor the permeability tensor. Instead the number and trend of the modeled grain boundaries by using the binseparate function revealed a strong impact on the effective $P$ wave velocities that varied from 3706 to $5043 \mathrm{~m} \mathrm{~s}^{-1}$ (Sell et al., 2013b). All results are depicted in Fig. 10, where a representative slice (no. 111) of each case is shown, revealing the effect of filtering and assignment of grain boundaries, as well as the rock characteristics. Comparing the $P$ wave velocity, porosity and permeability obtained by digital rock analysis with results from lab investigations performed on this Berea sample (Madonna et al., 2012), we found the bestmatching dataset to be Berlb filtered with NLM. Digital rock analysis indicated a total connected porosity of $18 \%$ and a permeability of $150 \mathrm{mD}$ for Ber $1 \mathrm{~b}$ where the lab results revealed a connected porosity of $\sim 20 \%$. The permeability of this sample, as provided by the Berea Sandstone ${ }^{\mathrm{TM}}$ Petroleum Cores (Ohio, USA), is between 200 and $500 \mathrm{mD}$. The worstmatching datasets during the determination of effective physical properties were the unfiltered and the median filtered one with a total porosity of $12 \%$ and a permeability of $80 \mathrm{mD}$ (Sell et al., 2013b).

\section{Wave propagation modeling in hydrate-bearing sedimentary matrices}

In this section, we present a first application of our derived post-processing data to wave propagation modeling. A series of numerical experiments based on segmented 3-D images of the structure has been conducted to obtain the $P$ wave velocity trend with increase or decrease of the hydrate bulk. This has been done to validate our model approach to aim for further wave propagation modeling including $S$ wave velocity and attenuation in future studies. As mentioned earlier, methane hydrate has been substituted by Xe hydrate that exhibits far reaching similarities in crystal structure and molecular size of guests (Table 1). Yet, in terms of the compressibility these clathrates are noticeably different; structures hosting Xe gas are about $10 \%$ stiffer than in the case of $\mathrm{CH}_{4}$ (Hansen et al., 2016). The difference originates in guest-host coupling which is strong for tightly fitting $\mathrm{Xe}$ and weaker for somewhat smaller methane that freely rattles in cages (Chazallon et al., 2002; Schober et al., 2003). Another potential difference might be found in the crystal size for both clathrates due to somewhat different density of nucleation sites. Yet, without a definite proof we must assume 


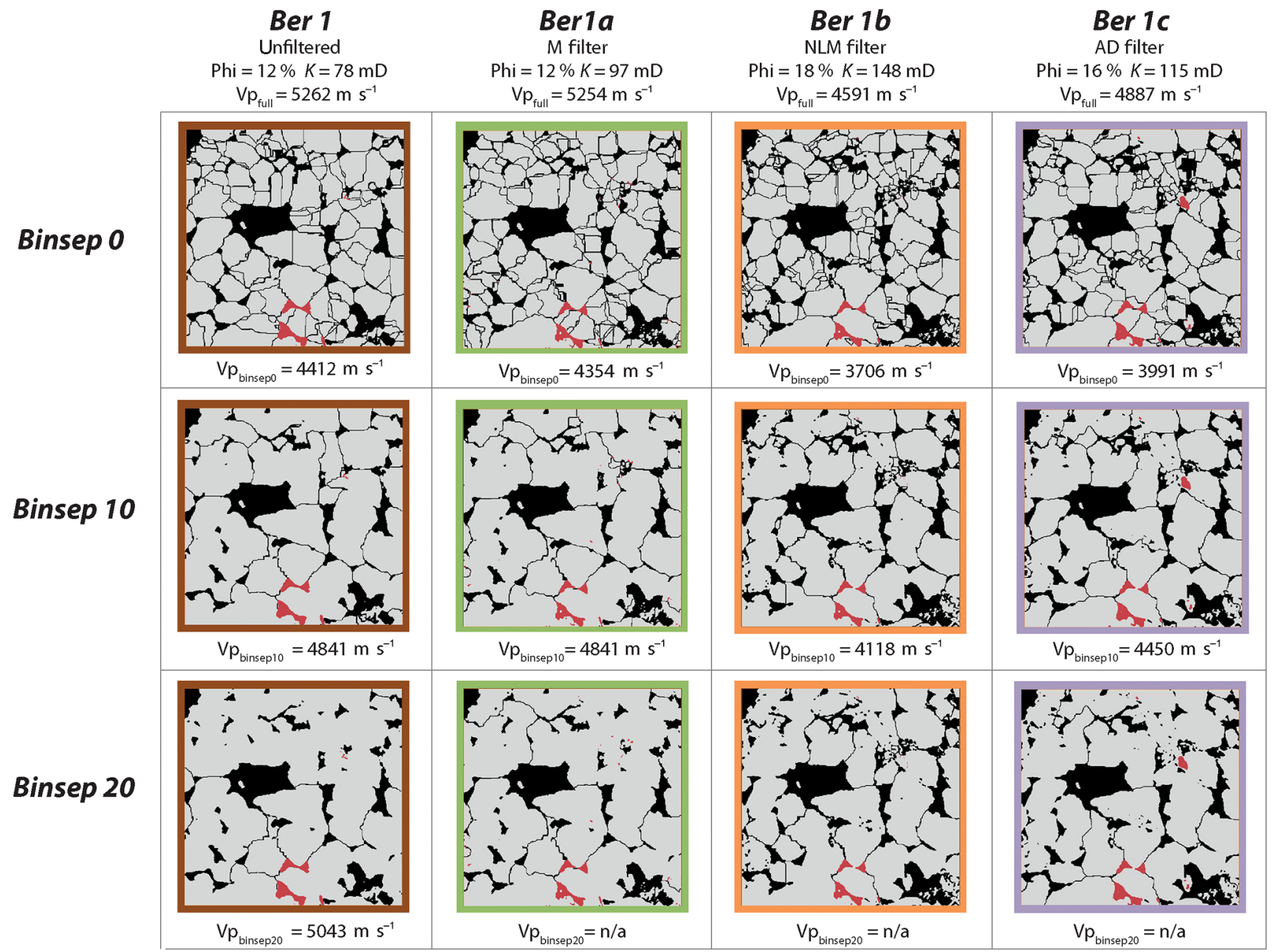

Figure 12. This figure comprises the results of the preliminary study on the effect of filtering on the modeling of effective elastic properties. Representative 2-D slices (\#111 of the $X-Y$ plane) are depicted.

for the moment that the hydrate nucleation and crystallization processes of both clathrates in the sedimentary matrix are comparable. We also take digitized Xe hydrate distribution and microstructure obtained from SRXCT and assign to it elastic moduli of $\mathrm{CH}_{4}$ hydrate: bulk modulus $(K)$, shear modulus $(G), P$ wave modulus $(M)$ and the density $(\rho)$.

We applied the rotated staggered finite-difference method appropriate for dynamic measurements. The fundamental idea of the model is discussed in detail by Saenger et al. (2004). This approach studies the wave propagation in heterogeneous materials within the long wavelength limit. Using this method it is possible to predict precisely effective elastic properties of various pore geometries in the relatively wide range between the upper and lower Hashin-Shtrikman bound. Complex structures, as observed in our samples, trigger strong scattering which can be treated only by numerical techniques since an analytical solution of the wave equation is not available. Finite difference (FD) methods discretize the wave equation on a grid and replace spatial derivatives by FD
Table 1. Comparison of crystallographic properties of xenon and methane hydrate (Hansen et al., 2016). Second part of the table depicts the elastic moduli later assigned for modeling the effective elastic properties. Values of $M$ ( $P$ wave modulus), $K$ (bulk modulus) and $G$ (shear modulus) (after Helgerud et al., 2003).

\begin{tabular}{llrr}
\hline & Xe hydrate & $\mathrm{CH}_{4}$ hydrate & Quartz \\
\hline Hydrate structure & sI & sI & \\
Cage diameter $(\AA)$ & $5.09-5.86$ & $5.1-5.85$ & \\
Lattice constant & 11.988 & 11.991 & \\
at 276 K $(\AA)$ & & & \\
Molecular size & 4.58 & 4.36 & \\
\hline$M(\mathrm{GPa})$ & & 13.57 & 96.98 \\
$K(\mathrm{GPa})$ & Substitution \\
$G(\mathrm{GPa})$ & of elastic \\
$\rho\left(\mathrm{kg} \mathrm{m}^{-3}\right)$ & densilis and \\
model & \\
\hline
\end{tabular}



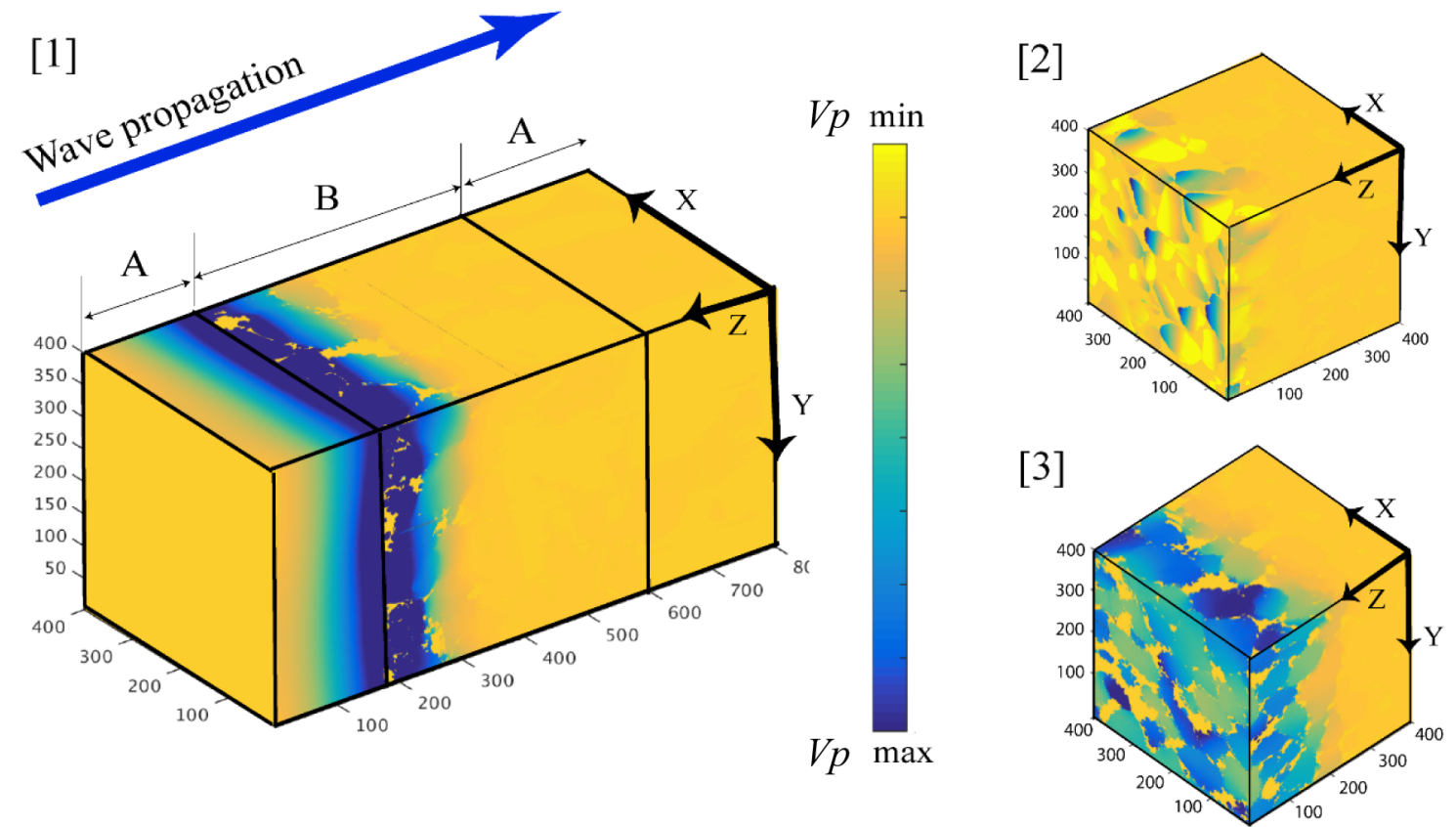

Figure 13. (1) Visualization of the RSG model where A is a predefined homogenous solid in which the $400 \times 400 \times 400$ size volume is embedded. The plane wave propagates in $z$ direction. (2) Visualization of the $V_{\mathrm{p}}$ in a sample containing roughly $7 \%$ of hydrate. (3) Visualization of the $V_{\mathrm{p}}$ in a sample bearing $17 \%$ of hydrate where the $V_{\mathrm{p}}$ is significantly higher due to the increased hydrate bulk.

operators using neighboring points. Instability problems on a staggered grid may be caused by discretization when the medium contains high-contrast discontinuities (e.g., pores or fractures). These difficulties can be avoided by using the rotated staggered grid technique proposed by Saenger and Bohlen (2004). Since the finite-difference approach is based on the wave equation without physical approximations, the method accounts not only for direct waves, primary and multiple reflected waves, but also for surface waves, head waves, converted reflected waves, and diffracted waves (Saenger et al., 2004).

Furthermore, the application of this method has already been benchmarked (Andrä et al., 2013a). On top of the 3-D rock model a body force plane source is applied using a homogeneous buffer zone of assigned vacuum (Sell et al., 2015). The plane wave travels through the embedded digitized 3-D sample volume (Fig. 13.1). Two plane receivers on the top and the bottom of the model measure the time delay of the plane wave's peak amplitude caused by the inhomogeneity of the rock (Madonna et al., 2013). As the model input is chosen to a volume of $400 \times 400 \times 400$ pixels due to computational limits, the segmented datasets needed to be reduced in size. This was accomplished by using the "resample" tool based on the Lanczos filter implemented in Avizo. Usually the default kernel Lanczos yields sufficiently good results for both minifications and magnifications. In the present case a cropped dataset of $1800 \times 1800 \times 1800$ voxels each of $(0.74 \mu \mathrm{m})^{3}$ in size was reduced to a cube of $400 \times 400 \times 400$ voxels each of $(3.4 \mu \mathrm{m})^{3}$ in size.

Effective velocities of the hydrate-bearing sedimentary matrices are determined by comparing the simulated results with those of a reference model. Note that the quartz grains have not been compacted during sample preparation wherein the hydrate is located in the pore space, which causes the structure to be stiffer with an increase in hydrate saturation (Fig. 13.2 and 13.3).

As the stop-and-go scanning procedure (Chaouachi et al., 2015; Falenty et al., 2015) permits a time-resolved observation of hydrate formation, the numerically obtained $P$ wave from the scans confirm the expected trend of an increase of $P$ wave velocities with higher hydrate bulk in the sediment (Priest et al., 2009). For each post-processed scan the $P$ wave velocity has been determined following the workflow described in this paper. In Fig. 13.2 the $P$ wave velocity simulated at the first time step is visualized for a sample with $7 \%$ of hydrate. The simulated $P$ wave velocity for a sample containing $17 \%$ of hydrate is visualized in Fig. 13.3. The comparison of both figures shows clearly how the $P$ wave velocity increases due to a higher hydrate bulk as the GH holds the quartz in place and stiffens the structure.

In Fig. 14, the results of the simulated $V_{\mathrm{p}}$ for series of eight scans are depicted. In absence of hydrate (scan 5) the lowest $P$ wave velocity of $1462 \mathrm{~ms}^{-1}$ is given for the model series. This $P$ wave value is typical for partially water-saturated sands, which is also reasonable. The highest $P$ wave veloci- 


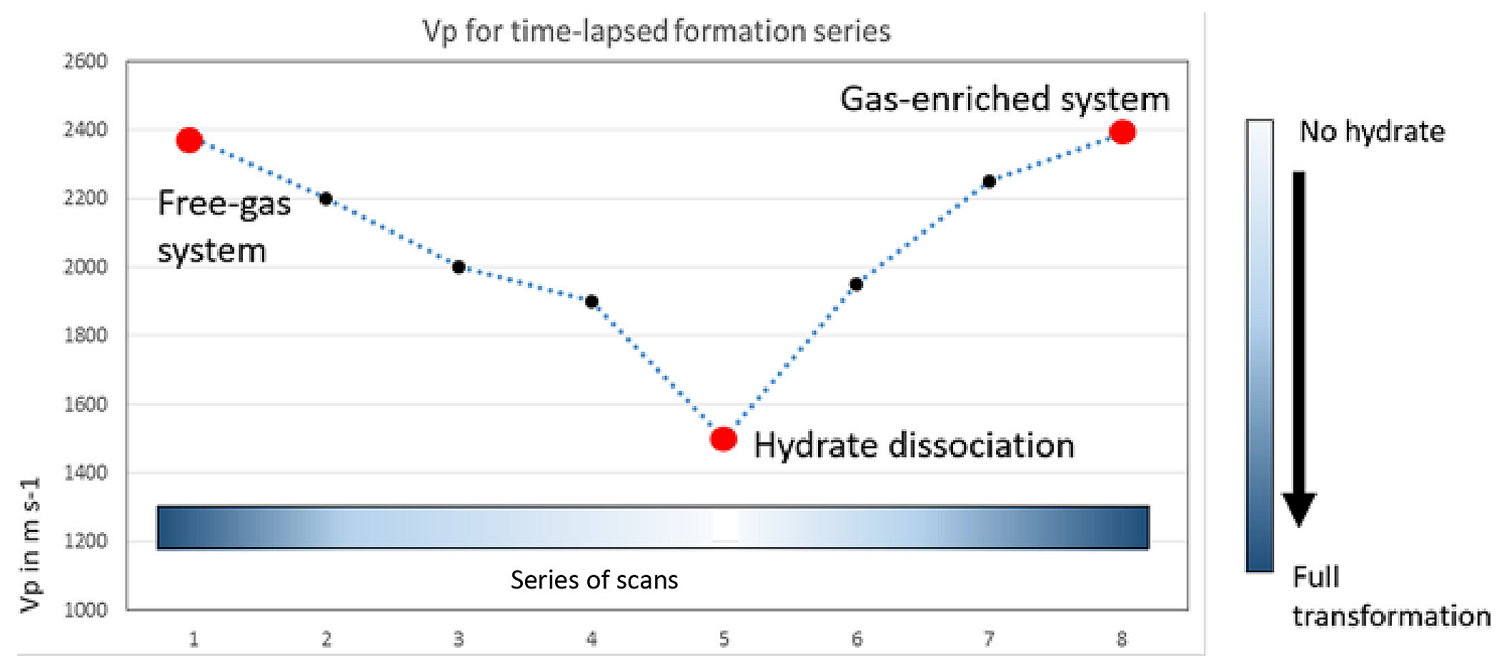

Figure 14. Results of the $p$ wave modeling of a series of scans starting at the initial state forming hydrate from a free gas system up to the full-transformed state, followed by a controlled destabilization of the system to maintain hydrate dissociation from which again the hydrate formation was triggered up to a full-transformed state in a gas-enriched system.

ties have been obtained for the full-transformed states as expected (scans 1 and 8). Scan 1 was taken when hydrate was formed from the free-gas system starting at the gas-water interface and scan 8 when hydrate was formed from a system where liquid water is metastable enriched with a gas (gasenriched system). For the first case the obtained $P$ wave velocity is $2368 \mathrm{~ms}^{-1}$ and for the second case it is $2408 \mathrm{~ms}^{-1}$. Therefore, at the full-transformed state of hydrate formed in a free-gas system (scan 1) the $P$ wave velocity is slightly lower than at the full-transformed state of hydrate formed in a gasrich system (scan 8), which has been discussed earlier in the literature (Priest et al., 2005; Konno et al., 2015; Waite et al., 2009). With the presented results of the modeled $P$ wave velocities the model approach is in a realistic range when compared with field (Carcione and Gei, 2004; Riedel et al., 2002; Yuan et al., 1996) and laboratory data (Zhang et al., 2011; Priest et al., 2005, 2009), but it is noteworthy that the modeled results give only slightly lower values than the experimental ones, even though at significantly lower hydrate saturation $(<20 \%)$ compared to results from laboratory work. Furthermore, intensive modeling on various scans needs to be performed to fully understand this apparently contrasting observation. In addition, one should take into account that investigations on seismic responses conducted in laboratory often use pre-compacted samples (Matsushima et al., 2015) while in our study we used unconsolidated samples. Moreover, there are uncertainties with the elastic response of $\mathrm{Xe}$ hydrate, likely to be somewhat different from the one of $\mathrm{CH}_{4}$ hydrate, which was taken for our analysis.

\section{Conclusions}

A novel processing approach, based on tomographic data with sub-micrometer scale resolution, is presented which allows one to obtain in the next step the effective elastic properties of hydrate-bearing sediments. This paper complements the paper of Chaouachi et al. 2015 with a sufficient workflow to process 3-D image data derived from synchrotronbased tomography to gain digitized results that are ready for subsequent use as a direct numerical model input. The data processing requires a pre-analysis to aim for best possible image quality enhancement. In our opinion, crucially important when aiming for multiphase 3-D imaging on the submicroscale, involving low-contrast materials, is the scanning itself and the image enhancement. Regarding the scanning part it is important to avoid scan artifacts, e.g., rotation errors which are not discussed in this paper but worth mentioning. The scanning procedure needs intensive testing prior to the experiment. All actions on image enhancement must be carried out with great care as the filters applied significantly alter the latter segmentation results as stated in the pre-analysis section of this paper. We highly recommend to carry out prestudies ahead of every digital rock physics approach where segmented data are needed for further modeling. Applying our proposed method it is possible to approximate the elastic properties of sediments containing less than $20 \%$ of hydrate within a realistic range at the sub-micrometer scale; a more detailed analysis and intercomparison is under way. As the time-resolved images produced a time series of hydrate formed from different scenarios (free-gas and gas-enriched), we are also able to distinguish between those cases. Yet, one should keep in mind that initially different microstructural settings (as a consequence of the different preparation routes) 
may well converge with time to closely similar distributions of gas hydrates in the sedimentary matrix (Chaouachi et al., 2015). Laboratory work usually is not converged when experiments are executed while natural samples are in general more mature. Ongoing work is focusing in more detail on the $P$ wave, $S$ wave velocities and attenuation since the presence of a thin fluid layer observed in the structures could explain the anomalous seismic responses of hydrate-bearing sediments observed in field studies. For this, a satisfying solution to segment the thin fluid layer in all scans is mandatory. In addition, we need to investigate further the contrasting observation of rather high results of $P$ wave velocities at low gas hydrate saturation.

Acknowledgements. We acknowledge the Paul Scherrer Institute, Villigen, Switzerland, for provision of synchrotron radiation beam time at the TOMCAT beamline of the SLS and would like to thank Bernd Pinzer for assistance. We are grateful to Jens-Oliver Schwarz, Martin Wolf and Faisal Khan (all formerly at JGU Mainz) for their valuable help during these experiments. The pressure cell was designed and constructed by Ulf Kahmann and Heiner Bartels (both from Göttingen). The financial support from the Deutsche Forschungsgemeinschaft (DFG grants Ke 508/20 and $\mathrm{Ku}$ 920/18) is gratefully acknowledged. Support also came from the $\mathrm{BMBF}$ in the framework of the SUGAR-II program (grant 03G0819B).

Edited by: S. Henkel

Reviewed by: M. Schindler and two anonymous referees

\section{References}

Andrä, H., Combaret, N., Dvorkin, J., Glatt, E., Han, J., Kabel, M., Keehm, Y., Krzikalla, F., Lee, M., Madonna, C., Marsh, M., Mukerji, T., Saenger, E. H., Sain, R., Saxena, N., Ricker, S., Wiegmann, A., and Zhan, X.: Digital rock physics benchmarks - Part II: Computing effective properties, Comput. Geosci., 50, 33-43, 2013a.

Andrä, H., Combaret, N., Dvorkin, J., Glatt, E., Han, J., Kabel, M., Keehm, Y., Krzikalla, F., Lee, M., Madonna, C., Marsh, M., Mukerji, T., Saenger, E. H., Sain, R., Saxena, N., Ricker, S., Wiegmann, A., and Zhan, X.: Digital rock physics benchmarks Part I: Imaging and segmentation, Comput. Geosci., 50, 25-32, 2013b.

Berg, S., Ott, H., Klapp, S. A., Schwing, A., Neiteler, R., Brussee, N., Makurat, A., Leu, L., Enzmann, F., Schwarz, J.-O., Kersten, M., Irvine, S., and Stampanoni, M.: Real-time 3d imaging of haines jumps in porous media flow, P. Natl. Acad. Sci. USA, 110, 3755-3759, 2013.

Berge, L. I., Jacobsen, K. A., and Solstad, A.: Measured acoustic wave velocities of $\mathrm{r} 11\left(\mathrm{ccl}_{3} \mathrm{f}\right)$ hydrate samples with and without sand as a function of hydrate concentration, J. Geophys. Res.Solid Earth, 104, 15415-15424, 1999.

Best, A., Priest, J., and Clayton, C.: A resonant column study of the seismic properties of methane-hydrate-bearing sand, in: Geophysical characterization of gas hydrates, edited by: Riedel, M.,
Willoughby, E. C., and Chopra, S., Society of Exploration Geophysicists, 337-347, 2010.

Best, A. I., Priest, J. A., Clayton, C. R. I., and Rees, E. V. L.: The effect of methane hydrate morphology and water saturation on seismic wave attenuation in sand under shallow sub-seafloor conditions, Earth Planet. Sc. Lett., 368, 78-87, 2013.

Bleau, A. and Leon, L. J.: Watershed-based segmentation and region merging, Comput. Vis. Image Und., 3, 317-370, 2000.

Boswell, R. and Collett, T. S.: Current perspectives on gas hydrate resources, Energ. Environ. Sci., 4, 1206-1215, 2011.

Buades, A., Coll, B., and Morel, J.-M.: A non-local algorithm for image denoising, IEEE Computer Society Conference on Computer Vision and Pattern Recognition, San Diego, CA, USA, 6065, 2005a.

Buades, A., Coll, B., and Morel, J. M.: A review of image denoising algorithms, with a new one, Multiscale Model. Sim., 4, 490-530, 2005b.

Carcione, J. M. and Gei, D.: Gas-hydrate concentration estimated from $p$ - and $s$-wave velocities at the mallik 21-38 research well, mackenzie delta, Canada, J. Appl. Geophys., 1, 73-78, 2004.

Chaouachi, M., Falenty, A., Sell, K., Enzmann, F., Kersten, M., Haberthür, D., and Kuhs, W. F.: Microstructural evolution of gas hydrates in sedimentary matrices observed with synchrotron x-ray computed tomographic microscopy, Geochem. Geophy. Geosy., 16, 1711-1722, 2015.

Chazallon, B., Itoh, H., Koza, M., Kuhs, W. F., and Schober, H.: Anharmonicity and guest-host coupling in clathrate hydrates, Phys. Chem. Chem. Phys., 4, 4809-4816, 2002.

Chuvilin, E. M., Istomin, V. A., and Safonov, S. S.: Residual nonclathrated water in sediments in equilibrium with gas hydrate comparison with unfrozen water, Cold Reg. Sci. Technol., 68, 68-73, 2011.

Cnudde, V. and Boone, M. N.: High-resolution x-ray computed tomography in geosciences: A review of the current technology and applications, Earth-Sci. Rev., 123, 1-17, 2013.

Dai, J., Xu, H., Snyder, F., and Dutta, N.: Detection and estimation of gas hydrates using rock physics and seismic inversion: Examples from the northern deepwater gulf of mexico, The Leading Edge, 23, 60-66, 2004.

Dai, S., Santamarina, J. C., Waite, W. F., and Kneafsey, T. J.: Hydrate morphology: Physical properties of sands with patchy hydrate saturation, J. Geophys. Res.-Solid Earth, 117, B11205, doi:10.1029/2012jb009667, 2012.

Desbois, G., Urai, J. L., PÉRez-Willard, F., Radi, Z., Offern, S., Burkart, I., Kukla, P. A., and Wollenberg, U.: Argon broad ion beam tomography in a cryogenic scanning electron microscope: A novel tool for the investigation of representative microstructures in sedimentary rocks containing pore fluid, J. Microscopy, 249, 215-235, 2013.

Deville, S., Adrien, J., Maire, E., Scheel, M., and Di Michiel, M.: Time-lapse, three-dimensional in situ imaging of ice crystal growth in a colloidal silica suspension, Acta Mater., 61, 20772086, 2013.

Dvorkin, J., Nur, A., Uden, R., and Taner, T.: Rock physics of a gas hydrate reservoir, The Leading Edge, 22, 842-847, 2003.

Ecker, C., Dvorkin, J., and Nur, A. M.: Estimating the amount of gas hydrate and free gas from marine seismic data, Geophysics, 65, 565-573, 2000. 
Falenty, A., Chaouachi, M., Neher, S. H., Sell, K., Schwarz, J.-O., Wolf, M., Enzmann, F., Kersten, M., Haberthur, D., and Kuhs, W. F.: Stop-and-go in situ tomography of dynamic processes gas hydrate formation in sedimentary matrices, Acta Crystallogr. A, 71, s154, doi:10.1107/S2053273315097740, 2015.

Guerin, G. and Goldberg, D.: Modeling of acoustic wave dissipation in gas hydrate-bearing sediments, Geochem. Geophy. Geosy., 6, Q07010, doi:10.1029/2005gc000918, 2005.

Hansen, T. C., Falenty, A., and Kuhs, W. F.: Lattice constants and expansivities of gas hydrates from $10 \mathrm{k}$ up to the stability limit, J. Chem. Phys., 144, 054301, doi:10.1063/1.4940729, 2016.

Helgerud, M. B., Waite, W. F., Kirby, S. H., and Nur, A.: Measured temperature and pressure dependence of vp and vs in compacted, polycrystalline si methane and sii methane-ethane hydrate, Can. J. Phys., 81, 47-53, 2003.

Hemes, S., Desbois, G., Urai, J. L., Schröppel, B., and Schwarz, J.O.: Multi-scale characterization of porosity in boom clay (hadeslevel, mol, belgium) using a combination of x-ray $\mu$-ct, $2 \mathrm{~d}$ bibsem and fib-sem tomography, Micropor. Mesopor. Mat., 208, 120, 2015.

Henke, B. L., Gullikson, E. M., and Davis, J. C.: X-ray interactions: Photoabsorption, scattering, transmission, and reflection at $e=50-30000 \mathrm{ev}, z=1-92$, Atom. Data Nucl. Data, 54, 181342,1993 .

Holzer, L. and Cantoni, M.: Review of fib-tomography, in: Nanofabrication using focused ion and electron beams: Principles and applications, edited by: Utke, I., Moshkalev, S. A., and Russell, P., Oxford University Press, NY, USA, 410-435, 2012.

Hu, G. W., Ye, Y. G., Zhang, J., Liu, C. L., Diao, S. B., and Wang, J. S.: Acoustic properties of gas hydrate-bearing consolidated sediments and experimental testing of elastic velocity models, J. Geophys. Res.-Solid Earth, 115, B02102, doi:10.1029/2008jb006160, 2010.

Iassonov, P., Gebrenegus, T., and Tuller, M.: Segmentation of x-ray computed tomography images of porous materials: A crucial step for characterization and quantitative analysis of pore structures, Water Resour. Res., 9, W09415, doi:10.1029/2009WR008087, 2009.

Jin, S., Nagao, J., Takeya, S., Jin, Y., Hayashi, J., Kamata, Y., Ebinuma, T., and Narita, H.: Structural investigation of methane hydrate sediments by microfocus $\mathrm{x}$-ray computed tomography technique under high-pressure conditions, Jpn. J. Appl. Phys., 2, L714-L716, 2006.

Kaestner, A., Lehmann, E., and Stampanoni, M.: Imaging and image processing in porous media research, Adv. Water Resour., 31, 1174-1187, 2008.

Kerkar, P. B., Horvat, K., Jones, K. W., and Mahajan, D.: Imaging methane hydrates growth dynamics in porous media using synchrotron x-ray computed microtomography, Geochem. Geophy. Geosy., 15, 4759-4768, 2014.

Klapp, S. A., Enzmann, F., Walz, P., Huthwelker, T., Tuckermann, J., Schwarz, J. O., Pape, T., Peltzer, E. T., Mokso, R., Wangner, D., Marone, F., Kersten, M., Bohrmann, G., Kuhs, W. F., Stampanoni, M., and Brewer, P. G.: Microstructure characteristics during hydrate formation and dissociation revealed by $\mathrm{x}$-ray tomographic microscopy, Geo-Mar. Lett., 32, 555-562, 2012.

Konno, Y., Jin, Y., Yoneda, J., Kida, M., Egawa, K., Ito, T., Suzuki, K., and Nagao, J.: Effect of methane hydrate morphology on compressional wave velocity of sandy sediments: Analysis of pressure cores obtained in the eastern nankai trough, Mar. Petrol. Geol., 66, 425-433, 2015.

Li, F. G., Sun, C. Y., Zhang, Q., Liu, X. X., Guo, X. Q., and Chen, G. J.: Laboratory measurements of the effects of methane/tetrahydrofuran concentration and grain size on the $p$ wave velocity of hydrate-bearing sand, Energ. Fuels, 25, 20762082, 2011.

Liu, Y., Kiss, A. M., Larsson, D. H., Yang, F., and Pianetta, P.: To get the most out of high resolution $x$-ray tomography: A review of the post-reconstruction analysis, Spectrochim. Acta B, 117, 29-41, 2016.

Madonna, C., Almqvist, B. S. G., and Saenger, E. H.: Digital rock physics: Numerical prediction of pressure-dependent ultrasonic velocities using micro-ct imaging, Geophys. J. Int., 3, 14751482, 2012.

Madonna, C., Quintal, B., Frehner, M., Almqvist, B. S. G., Tisato, N., Pistone, M., Marone, F., and Saenger, E. H.: Synchrotronbased x-ray tomographic microscopy for rock physics investigations, Geophysics, 78, 53-64, doi:10.1190/geo2012-0113.1, 2013.

Marone, F. and Stampanoni, M.: Regridding reconstruction algorithm for real-time tomographic imaging, J. Synchrotron Radiat., 19, 1029-1037, 2012.

Matsushima, J., Suzuki, M., Kato, Y., and Rokugawa, S.: Ultrasonic compressional and shear attenuation measurements in partially frozen consolidated and unconsolidated porous media, J. Geophys. Res., 81, 141-153, 2015.

Moridis, G. J., Collett, T. S., Pooladi-Darvish, M., Hancock, S., Santamarina, C., Boswell, R., Kneafsey, T., Rutqvist, J., Kowalsky, M. B., Reagan, M. T., Sloan, E. D., Sum, A. K., and Koh, C. A.: Challenges, uncertainties, and issues facing gas production from gas-hydrate deposits, SPE Reserv. Eval. Eng., 14, 76-112, 2011.

Murshed, M. M., Klapp, S. A., Enzmann, F., Szeder, T., Huthwelker, T., Stampanoni, M., Marone, F., Hintermuller, C., Bohrmann, G., Kuhs, W. F., and Kersten, M.: Natural gas hydrate investigations by synchrotron radiation $x$-ray cryotomographic microscopy (srxctm), Geophys. Res. Lett., 35, L23612, doi:10.1029/2008g1035460, 2008.

Paganin, D., Mayo, S. C., Gureyev, T. E., Miller, P. R., and Wilkins, S. W.: Simultaneous phase and amplitude extraction from a single defocused image of a homogeneous object, J. Microsc.Oxford, 206, 33-40, 2002.

Pak, T., Butler, I. B., Geiger, S., van Dijke, M. I. J., and Sorbie, K. S.: Droplet fragmentation: 3d imaging of a previously unidentified pore-scale process during multiphase flow in porous media, P. Natl. Acad. Sci. USA, 112, 1947-1952, 2015.

Porter, M. L. and Wildenschild, D.: Image analysis algorithms for estimating porous media multiphase flow variables from computed microtomography data: A validation study, Computat. Geosci., 14, 15-30, 2010.

Priest, J. A., Best, A. I., and Clayton, C. R. I.: A laboratory investigation into the seismic velocities of methane gas hydratebearing sand, J. Geophys. Res.-Solid Earth, 110, B04102, doi:10.1029/2004jb003259, 2005.

Priest, J. A., Best, A. I., and Clayton, C. R. I.: Attenuation of seismic waves in methane gas hydrate-bearing sand, Geophys. J. Int., 164, 149-159, 2006. 
Priest, J. A., Rees, E. V. L., and Clayton, C. R. I.: Influence of gas hydrate morphology on the seismic velocities of sands, J. Geophys. Res.-Solid Earth, 114, B11205, doi:10.1029/2009jb006284, 2009.

Ramponi, G.: Warped distance for space-variant linear image interpolation, IEEE Transact. Image Proc., 5, 629-639, 1999.

Reza, A. M.: Realization of the contrast limited adaptive histogram equalization (clahe) for real-time image enhancement, J. VLSI Sig. Proc. Syst., 38, 35-44, 2004.

Riedel, M., Spence, G. D., Chapman, N. R., and Hyndman, R. D.: Seismic investigations of a vent field associated with gas hydrates, offshore vancouver island, J. Geophys. Res.-Solid Earth, 107, 2200, doi:10.1029/2001JB000269, 2002.

Saenger, E. H., Enzmann, F., Keehm, Y., and Steeb, H.: Digital rock physics: Effect of fluid viscosity on effective elastic properties, J. Appl. Geophys., 4, 236-241, 2011.

Saenger, E. H. and Bohlen, T.: Finite-difference modeling of viscoelastic and anisotropic wave propagation using the rotated staggered grid, Geophysics, 69, 583-591, 2004.

Saenger, E. H., Sell K., and Kersten, M.: On the Effect of Image Enhancement Techniques on Digital Rock Physics Results, 75th EAGE Conference \& Exhibition incorporating SPE EUROPEC 2013, Extended Abstract, London, UK, 2013.

Saenger, E. H., Krüger, O. S., and Shapiro, S. A.: Numerical considerations of fluid effects on wave propagation: Influence of the tortuosity, Geophys. Res. Lett., 31, L21613, doi:10.1029/2004g1020970, 2004.

Sarker, S., Chowdhury, S., Laha, S., and Dey, D.: Use of non-local means filter to denoise image corrupted by salt and pepper noise, SIPIJ Signal \& Image Processing: An International Journal, 3, 223-235, 2012.

Schober, H., Itoh, H., Klapproth, A., Chihaia, V., and Kuhs, W. F.: Guest-host coupling and anharmonicity in clathrate hydrates, Eur. Phys. J. E, 12, 41-49, 2003.

Sell, K., Enzmann, F., Spangenberg, E., and Kersten, M.: $\mathrm{CO}_{2}$ injection to a saline aquifer sandstone - clay mineral displacement and permeability changes, 75th EAGE Conference \& Exhibition incorporating SPE EUROPEC 2013, Extended Abstract, London, UK, 2013a.

Sell, K., Madonna, C., Quintal, B., Frehner, M., Tisato, N., and Saenger, E. H.: Synchrotron-based x-ray tomographic images and segmentation techniques to account for effects of grain contacts and micro-cracks on rock properties, SBGf International Congress, Rio de Janeiro, Brazil, 26-29 August, $2013 \mathrm{~b}$.

Sell, K., Chaouachi, M., Falenty, A., Saenger, E. H., Khan, F., Schwarz, J.-O., Enzmann, F., Kersten, M., and Kuhs, W. F.: Microstructure of hydrate-bearing sediments and determination of p-wave velocities based on high-resolution synchrotron tomographic data, SEG Technical Meeting and Conference, New Orleans, LA, USA, 2015.
Shulakova, V., Pervukhina, M., Müller, T. M., Lebedev, M., Mayo, S., Schmid, S., Golodoniuc, P., De Paula, O. B., Clennell, M. B., and Gurevich, B.: Computational elastic up-scaling of sandstone on the basis of x-ray micro-tomographic images, Geophys. Prospect., 61, 287-301, 2013.

Sloan, E. D. and Koh, C. A.: Clathrate hydrates of natural gases, 3th Edn., Vol. 119, CRC Press, Boca Raton, Fla, 2008.

Spangenberg, E. and Kulenkampff, J.: Influence of methane hydrate content on electrical sediment properties, Geophys. Res. Lett., 33, L24315, doi:10.1029/2006g1028188, 2006.

Stampanoni, M., Groso, A., Isenegger, A., Mikuljan, G., Chen, Q., Bertrand, A., Henein, S., Betemps, R., Frommherz, U., Böhler, P., Meister, D., Lange, M., and Abela, R.: Trends in synchrotronbased tomographic imaging: The sls experience, SPIE Proceedings, 2006,

Vincent, L. and Soille, P.: Watersheds in digital spaces: An efficient algorithm based on immersion simulations, IEEE T. Pattern Anal., 6, 583-598, 1991.

Waite, W. F., Winters, W. J., and Mason, D. H.: Methane hydrate formation in partially water-saturated ottawa sand, Am. Mineral., 89, 1202-1207, 2004.

Waite, W. F., Santamarina, J. C., Cortes, D. D., Dugan, B., Espinoza, D. N., Germaine, J., Jang, J., Jung, J. W., Kneafsey, T. J., Shin, H., Soga, K., Winters, W. J., and Yun, T. S.: Physical properties of hydrate-bearing sediments, Rev. Geophys., 47, RG4003, doi:10.1029/2008rg000279, 2009.

Wang, D.: A multiscale gradient algorithm for image segmentation using watersheds, Pattern Recogn., 12, 2043-2052, 1997.

Wang, J.-Q., Zhao, J.-F., Yang, M.-J., Li, Y.-H., Liu, W.-G., and Song, Y.-C.: Permeability of laboratory-formed porous media containing methane hydrate: Observations using X-ray computed tomography and simulations with pore network models, Fuel, $145,170-179,2015$.

Wang, J., Zhao, J., Zhang, Y., Wang, D., Li, Y., and Song, Y.: Analysis of the effect of particle size on permeability in hydratebearing porous media using pore network models combined with ct, Fuel, 136, 34-40, 2016.

Yuan, T., Hyndman, R. D., Spence, G. D., and Desmons, B.: Seismic velocity increase and deep-sea gas hydrate concentration above a bottom-simulating reflector on the northern cascadia continental slope, J. Geophys. Res.-Solid Earth, 101, 1365513671, 1996.

Yun, T. S., Francisca, F. M., Santamarina, J. C., and Ruppel, C.: Compressional and shear wave velocities in uncemented sediment containing gas hydrate, Geophys. Res. Lett., 32, L10609, doi:10.1029/2005g1022607, 2005.

Zhang, Q., Li, F. G., Sun, C. Y., Li, Q. P., Wu, X. Y., Liu, B., and Chen, G. J.: Compressional wave velocity measurements through sandy sediments containing methane hydrate, Am. Mineral., 96, 1425-1432, 2011. 